\title{
Nonlinear variability of body sway in patients with phobic postural vertigo
}

\section{Roman Schniepp ${ }^{1,2 t}$, Max Wuehr ${ }^{2}{ }^{+}{ }^{\dagger}$, Cauchy Pradhan ${ }^{2}$, Sergej Novozhilov ${ }^{1}$, Siegbert Krafczyk ${ }^{1}$, Thomas Brandt ${ }^{2,3}$ and Klaus Jahn ${ }^{1,2}$}

1 Department of Neurology, University of Munich, Munich, Germany

${ }^{2}$ German Center for Vertigo and Balance Disorders, University of Munich, Munich, Germany

${ }^{3}$ Institute of Clinical Neurosciences, University of Munich, Munich, Germany

Edited by:

Stefano Ramat, Università degli Studi di Pavia, Italy

\section{Reviewed by:}

Alexander A. Tarnutzer, University Hospital Zurich, Switzerland

Marcos Duarte, Federal University of $A B C$, Brazil

\section{${ }^{*}$ Correspondence:}

Max Wuehr, Department of Neurology, German Center for Vertigo and Balance Disorders, University of Munich, Marchioninistrasse 15, 81377 Munich, Germany

e-mail:mail@maxw.de

${ }^{\dagger}$ Roman Schniepp and Max Wuehr have contributed equally to this work.
Background: Subjective postural imbalance is a key symptom in the somatoform phobic postural vertigo (PPV). It has been assumed that more attentional control of body posture and / or co-contraction of leg muscles during standing is used to minimize the physiological body sway in PPV. Here we analyze nonlinear variability of body sway in patients with PPV in order to disclose changes in postural control strategy associated with PPV.

Methods: Twenty patients with PPV and 20 age-matched healthy subjects (HS) were recorded on a stabilometer platform with eyes open (EO), eyes closed (EC), and while standing on a foam rubber with eyes closed (ECF). Spatio-temporal changes of the center of pressure (CoP) displacement were analyzed to assess the structure of postural variability by computing the scaling exponent $\alpha$ and the sample entropy (SEn) of the time series.

Results: With EO on firm ground $\alpha$ and SEn of CoP displacement were significantly lower in patients $(p<0.001)$. For more difficult conditions (EC, ECF) postural variability in PPV assimilated to that of HS.

Conclusion: Postural control in PPV patients differs from HS under normal stance condition. It is characterized by a reduced scaling behavior and higher regularity. These changes in the structure of postural variability might suggest an inappropriate attentional involvement with stabilizing strategies, which are used by HS only for more demanding balance tasks.

Keywords: stance, psychogenic, vertigo, sample entropy, DFA, scaling exponent

\section{INTRODUCTION}

Phobic postural vertigo (PPV) has been described as a syndrome of subjective postural and gait instability in combination with a non-rotational vertigo or dizziness, mainly affecting patients with an obsessive-compulsive personality (1). PPV is one of the primary and secondary somatoform dizziness syndromes (2-4) and is also termed visual vertigo syndrome (5) or chronic subjective dizziness (6). As one of the most frequent causes of chronic dizziness, it has a high impact on functioning and quality of life (2, 7). The diagnostic criteria mainly comprise anxiety related symptoms in combination with normal otoneurological testing (1). However objective tests for the positive diagnosis of PPV are not available.

Body sway recording has been suggested to be of value in PPV $(8,9)$. Postural control is commonly assessed by analyzing amplitude parameters such as sway amplitude and sway path length. However, these parameters do not reveal information about the variability of sway, which is an important feature of motor control (10). Postural behavior is characterized by strong nonlinearities due to elastic and damping properties of muscles and nonlinear feedback control $(11,12)$. Analytic approaches from nonlinear systems theory seem promising for describing the structure of postural variability and have been used in several studies (13-16). Moreover, it has been suggested that nonlinear measures more reliably quantify body sway than traditional amplitude-related measures (17).

It has been hypothesized that altered postural behavior in patients with PPV is caused by an inappropriate postural strategy in which patients exert extensive supraspinal control in particular during normal stance conditions (18-20). In contrast, healthy balance control is mainly achieved by automated mechanisms not involving conscious control (21). Increased alertness is required for unusual and non-adapted balance tasks, which bare the risk of falls. Attentional postural control influences the structure of body sway variability (14), which can be quantified by the analysis of the scaling exponent $\alpha$ and the sample entropy (SEn). Both measures provide information related to the underlying motor control strategies governing postural stability. The scaling exponent $\alpha$ examines the presence of long-range correlations within the time series of the center of pressure (CoP) trajectories. The presence of long-range correlations implies that the postural sway behavior at any given point of time depends on the global history of the $\mathrm{CoP}$ time series: the stronger the long-range correlations within $\mathrm{CoP}$ time series, the more constrained the overall postural 
sway behavior (22). In addition, SEn quantifies the regularity or predictability of the CoP time series.

The purpose of this study was to investigate stance behavior in patients with PPV by analyzing the structure of postural variability, which has been shown to reveal motor control strategies governing postural control. The major question of the study was whether there are changes in the structure of body sway variability in patients with PPV that may indicate that these patients apply postural control strategies that are inappropriate with respect to normal balance conditions. Moreover, we aimed to see whether postural strategies change under more demanding balance tasks, like standing with eyes closed (EC) or on a foam rubber.

\section{MATERIALS AND METHODS SUBJECTS}

Twenty patients with PPV (14 females; mean age $38.4 \pm 13.8$ years; mean height: $1.75 \pm 0.11 \mathrm{~m}$; mean weight: $72.4 \pm 8.3 \mathrm{~kg}$ ) and 20 age-matched asymptotic healthy subjects (HS) ( 8 females; mean age $37.1 \pm 10.6$ years; mean height: $1.78 \pm 0.12 \mathrm{~m}$; mean weight: $74.5 \pm 7.5 \mathrm{~kg}$ ) participated in the study. The diagnosis of PPV was based on the diagnostic criteria suggested by Brandt (1). Mean duration of the symptoms was $1.3 \pm 0.6$ years. Patients underwent a standardized diagnostic procedure: Afferent somatosensory deficits were excluded by vibrotactile and proprioceptive assessment. Vestibular testing included a caloric irrigation of the horizontal semicircular canals $\left(30 / 44^{\circ} \mathrm{C}\right)$ and the head impulse testing of the horizontal vestibulo-ocular reflex (23). These procedures revealed normal somatosensory and vestibular functions in all participants. Anxiety was reported or admitted on direct questioning by eight patients (40\%), whereas vegetative symptoms such as palpitations, sweating, dyspnea, or diarrhea were only present in two patients (10\%). A history of coexisting panic disorder and/or agoraphobia was obtained in only one patient (5\%), whereas four patients $(20 \%)$ had a confirmed history of depression or anxiety disorder. Nine patients (45\%) reported an avoidance behavior of triggering situations, i.e., open spaces, closed places like elevators, crowds, or bridges. None of the patients admitted a regular use of benzodiazepines, antidepressants, or other neuromodulatory drugs. Six patients received physiotherapeutic interventions in history. However, a continued balance training, which could affect the posturography measurements, was not undertaken by any of the participants.

All subjects gave their written informed consent prior to the experiments. The study protocol was approved by the local Ethics Committee. The study was conducted in conformity with the Declaration of Helsinki.

\section{MEASUREMENT OF POSTURAL SWAY}

Static postural control was measured during upright stance on a stabilometer platform (Type 9261 A; Kistler, Winterthur, Switzerland), which transduces force changes exerted on foot support at a sampling frequency of $40 \mathrm{~Hz}$. Subjects were instructed to remain quiet upright stance (feet splayed at an angle of $30^{\circ}$, arms in hanging position) to omit any voluntary movements during the recordings. The postural control of each subject was recorded for three different stance conditions, standing with eyes open (EO), standing with EC, and standing with eyes closed on a foam rubber
(ECF). Each stance condition was recorded for $30 \mathrm{~s}$. The recorded signals were bidirectionally filtered (second-order low-pass Butterworth filter, cut-off frequency of $12.5 \mathrm{~Hz}$ ) to eliminate low amplitude measurement noise (14). Then the displacement of $\mathrm{CoP}$ in the medial-lateral (ML) and the anterior-posterior (AP) directions were calculated (Figure 1). All analysis was done using Matlab (The Mathworks, Version 2011a).

\section{DATA ANALYSIS}

Nonlinear measures were applied to estimate the structure of postural variability, i.e., the scaling behavior (scaling exponent $\alpha$ ) and the signal regularity (SEn) of the CoP time series.

\section{Scaling exponent}

The scaling exponent $\alpha$ was calculated by using a fractal analysis method for biological time signals called detrended fluctuation analysis (DFA) (24). In a first step, the mean is subtracted from the original time series, which is then integrated:

$$
y(k)=\sum_{i=1}^{k}[x(i)-\bar{x}]
$$

This integration step transforms the bounded series (i.e., CoP series) into an unbounded series, which has been suggested to avoid shortcomings of earlier methods (25). The integrated series is then divided into windows of equal length $n$ ranging from 4 to $N / 4$ data points. The local trend of each window $y_{n}$ is obtained and subtracted from the summed series by using a least-squared fit to obtain the detrended fluctuation $F(n)$ :

$$
F(n)=\sqrt{\frac{1}{N} \sum_{k=1}^{N}\left[y(k)-y_{n}\right]^{2}}
$$

The fluctuation of the detrended series, $F(n)$, is characterized by the power law $F(n) \propto n^{\alpha}$, where $\alpha$ is the slope of a double logarithm plot of $F(n)$ vs. $n$ (Figure 2). The scaling exponent $\alpha$ gives a quantitative measure for the strength of long-range correlations within the time series. A scaling exponent $\alpha=0.5$ indicates uncorrelated data (i.e., white noise); $\alpha$ values between 0.5 and 1.0 indicate persistent long-range power-law correlations; the closer $\alpha$ is to 1.0 the greater the influence of the distant past when compared with the influence of the recent past. The case of $\alpha=1.0$ corresponds to $1 / f$ noise, where present events are approximately equally correlated with events from the recent and the very distant past. For $\alpha>1.0$ correlations exist but cease to be of a power-law form; $\alpha=1.5$ indicates brown noise, i.e., integrated white noise. Brown noise is influenced by the recent past much more strongly than by the distant past and is therefore characterized by only local correlations $(24,26,27)$.

\section{Sample entropy}

The signal regularity was quantified using the SEn analysis (28, 29). The SEn analysis indexes the regularity of a time series by calculating the probability that having repeated itself for a window length $m$ within a tolerance of $r$, will also repeat itself for $m+1$ data points, without allowing self-matches. Thereby, lower values of SEn are associated with higher regularity of the time series, 
A

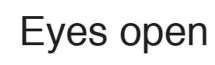

B Eyes closed
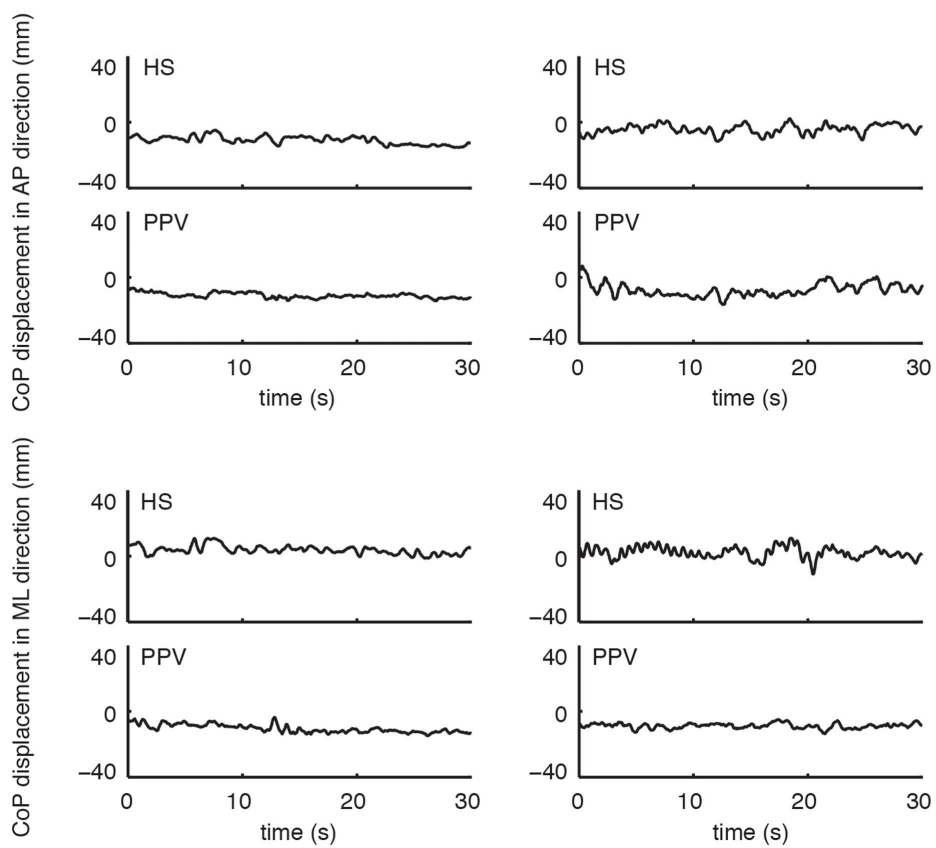

c Eyes closed \& foam
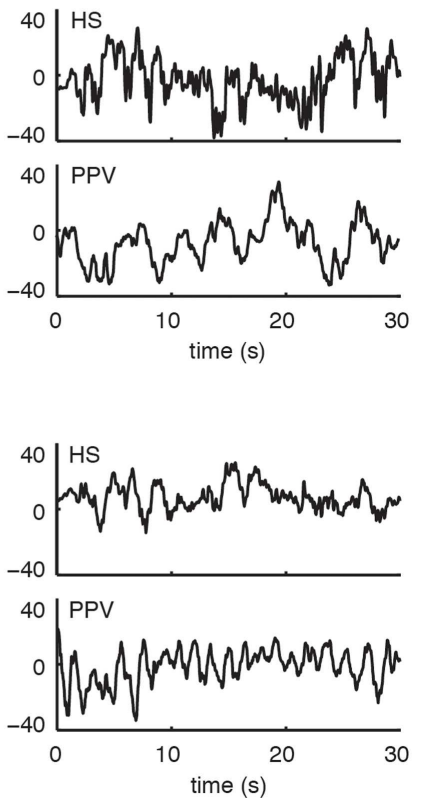

FIGURE 1 | Example of time series of center of pressure displacement in the anterior-posterior (AP) direction (upper panel) and the medial-lateral (ML) direction (lower panel) for one patient with phobic postural vertigo (PPV) and one healthy subject (HS) with eyes open (A), eyes closed (B), and eyes closed while standing on a foam (C)

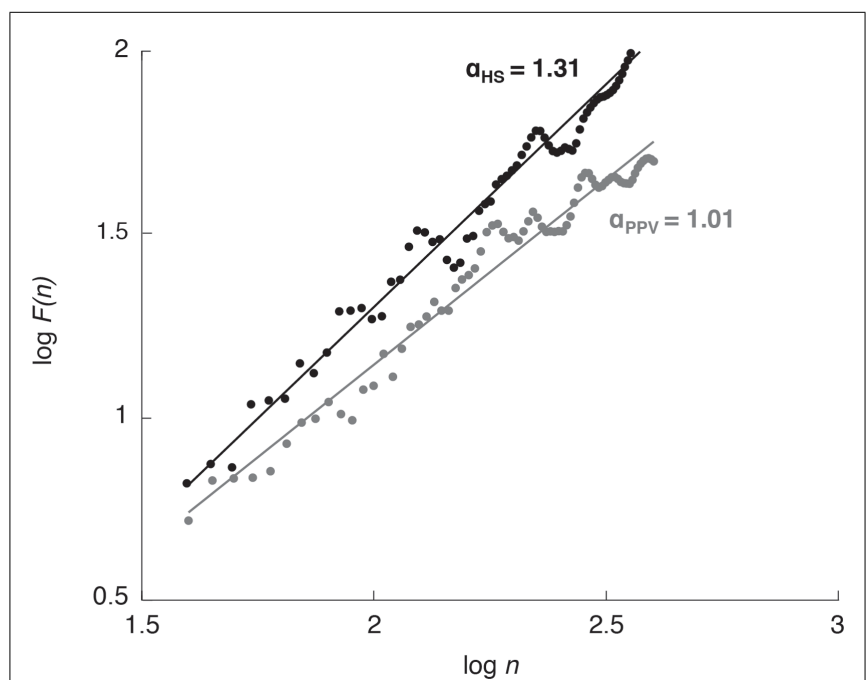

FIGURE 2 | Detrended fluctuation analysis plot for one healthy subject (HS; black dots and line) and one patient with phobic postural vertigo (PPV; gray dots and line) while standing with eyes open. The scaling exponent $\alpha$ quantifies the strength of long-range correlations within the CoP time series.

which means a greater likelihood that sets of matching epochs in a time series will be followed by another match within a certain tolerance.
Prior data processing comprised the computation of the increment of the recorded CoP time series according to the suggestions of Govindan et al. (30). The increment data was simply obtained by computing the differences between consecutive CoP values, thereby removing non-stationarity of the original CoP data. After the preceding data processing, SEn values were computed of the increment time series with input parameters $m$ and $r$ by using the PhysioToolkit-PhysioNetSampEn software (31). Parameters $m$ and $r$ were chosen according to the procedure described in Ramdani et al. (32), obtaining the value $m=3$ and $r=0.4$ for both ML and AP directions.

\section{STATISTICAL ANALYSIS}

The effects for each dependent variable were analyzed using a twoway ANOVA with group and condition as factors. Post hoc analysis was carried out using a Bonferroni post hoc test and results are reported significant if $p<0.05$. Statistical analysis was performed using SPSS (Version 20).

\section{RESULTS}

The results of the two-way ANOVA are presented in Table 1.

\section{SCALING EXPONENT $\alpha$}

For the CoP displacement in the ML direction, no significant differences of $\alpha$ between PPV patients and HS could be detected as reflected in the mean values for $\mathrm{EO}$ (PPV vs. HS: $1.13 \pm 0.19$ vs. $1.22 \pm 0.17$ ), for EC (PPV vs. HS: $1.03 \pm 0.21$ vs. $1.14 \pm 0.13$ ) and for ECF (PPV vs. 
Table 1 | Results of the two-way ANOVA on the scaling exponent $\alpha$ and SEn of the CoP displacement in the medial-lateral (ML) and the anterior-posterior (AP) plane.

Group (PPV/HS)

Condition (EO/EC/ECF)
$F_{2,40}=3.5, p=0.034$

$F_{2,40}=39.2, p<0.001$
Group $x$ condition

\section{DIRECTION}

Scaling exponent

Sample entropy

$F_{1,40}=2.3, p=0.129$

$F_{1,40}=33.8, p<0.001$

$F_{2,40}=1.4, p=0.002$

$F_{1,40}=5.8, p<0.001$

$F_{2,40}=34.1, p=0.001$

$F_{1,40}=30.0, p<0.001$

Scaling exponent

Significant differences are marked bold.

PPV, patients with phobic postural vertigo; HS, healthy subjects; EO, eyes open; EC, eyes closed; ECF, eyes closed and standing on foam.

HS: $1.11 \pm 0.18$ vs. $1.07 \pm 0.17)$. In HS, $\alpha$ was significantly lower for the condition ECF compared to EO (Bonferroni, $p=0.009$ ) (Figure 3A).

For the CoP displacement in the AP direction, $\alpha$ of patients compared to HS was significantly lower for the condition EO (PPV vs. HS: $1.05 \pm 0.19$ vs. $1.31 \pm 0.08 ; p<0.001$ ), and for EC (PPV vs. HS: $0.99 \pm 0.20$ vs. $1.13 \pm 0.09 ; p=0.007)$. During standing under ECF no significant differences between the groups were found (PPV vs. HS: $1.09 \pm 0.09$ vs. $1.11 \pm 0.16 ; p>0.05$ ). In HS, the scaling exponent $\alpha$ was significantly lower for the condition EC as well as for the condition ECF compared to EO (Bonferroni, for both $p<0.001)$. No significant differences of $\alpha$ between the standing conditions were found in PPV patients (Figure 3B).

\section{SAMPLE ENTROPY}

For the CoP displacement in the ML direction, SEn of PPV patients compared to HS was significantly lower for all conditions. The mean values were for EO (PPV vs. HS: $1.13 \pm 0.21$ vs. $1.30 \pm 0.15 ; p=0.006$ ), for $\mathrm{EC}$ (PPV vs. HS: $0.91 \pm 0.24$ vs. $1.23 \pm 0.21 ; p<0.001)$ and for ECF (PPV vs. HS: $0.79 \pm 0.14$ vs. $0.90 \pm 0.18 ; p=0.035)$. Within the HS group, SEn of the CoP displacement in the ML direction was significantly lower for the condition ECF compared to EO and compared to EC (Bonferroni, both $p<0.001)$. In patients with PPV, SEn in the ML direction was significantly lower for the condition $\mathrm{EC}$ as well as for the condition ECF compared to EO [Bonferroni, $p=0.011$ (EC vs. EO) and $p<0.001$ (ECF vs. EO)] and significantly lower for the condition ECF compared to EC (Bonferroni, $p<0.001$ ) (Figure 4A).

For the CoP displacement in the AP direction, SEn of PPV patients was significantly lower for the conditions EO and EC compared to HS. Mean values were for EO (PPV vs. HS: $0.88 \pm 0.23$ vs. $1.19 \pm 0.20 ; p<0.001$ ), for EC (PPV vs. HS: $0.73 \pm 0.24$ vs. $0.98 \pm 0.23 ; p=0.001$ ) and for ECF (PPV vs. HS: $0.61 \pm 0.15$ vs. $0.70 \pm 0.19 ; p>0.05)$. Within the HS group, SEn of the CoP displacement pattern was significantly lower for the condition EC as well as for the condition ECF compared to EO [Bonferroni, $p=0.007$ (EC vs. EO) and $p<0.001$ (ECF vs. EO)] and for ECF compared to EC (Bonferroni, $p<0.001$ ). Within the PPV group, SEn in the AP direction was significantly lower for the condition EC as well as for the condition ECF compared to EO [Bonferroni, $p=0.003$ (EC vs. EO) and $p<0.001$ (ECF vs. EO)] and significantly lower for the condition ECF compared to EC (Bonferroni, $p<0.001$ ) (Figure 4B).

\section{DISCUSSION}

The results of the study show that during normal balance condition postural control in PPV is characterized by a less complex, i.e., more regular and constrained mode of standing compared to HS. This difference largely disappears for more demanding balance tasks. The results can be interpreted in the way that PPV patients use an inappropriate balance strategy at baseline, which is only used by healthy controls for difficult and demanding balance tasks.

\section{COMPLEXITY IN POSTURAL CONTROL IN PPV}

Healthy postural control under normal stance conditions exhibits highly irregular, complex dynamics that represent interacting regulatory processes, which operate on different time scales (14). Such processes enable the postural system to prepare for reacting to sudden balancing stresses and thereby enhance the overall stability of a standing subject. When the postural control system is perturbed the complex steady-state dynamics may transiently change to a less complex mode of reactive tuning, which is characterized by closedloop responses that act on relatively short time periods to restore the balance equilibrium (33). An overall decrease of the inherent complexity in the steady-state dynamics is associated with a functional decline of the postural control system resulting in maladaptive responses to perturbations and thereby destabilizing the balance control (34).

Under unperturbed stance conditions postural control in PPV patients in AP direction was characterized by a decreased scaling exponents $\alpha$ close to $1 / f$ noise, indicating an increase in strength of long-range correlations. The relatively stronger dependency between the time scales in the CoP signal indicates that a smaller number of independently controllable system elements contribute to the motor output $(22,35,36)$. The increased strength of longrange correlations in the sway pattern of patients with PPV may imply a highly constrained postural behavior and decreased local stability (14) in contrast to the flexible and readily adaptable (with respect to changing balance conditions) healthy mode of standing (33). For HS, increasing the demands of the balance task (i.e., EC, ECF) led to a successive decrease of $\alpha$ toward $1 / f$ noise (in ML and AP directions) in accordance to previous studies $(14-17,35)$. In contrast, the scaling exponent $\alpha$ of patients with PPV did not decrease further and was similar to HS under the same condition. This supports the hypothesis that PPV patients use a strategy at baseline that is used normally 

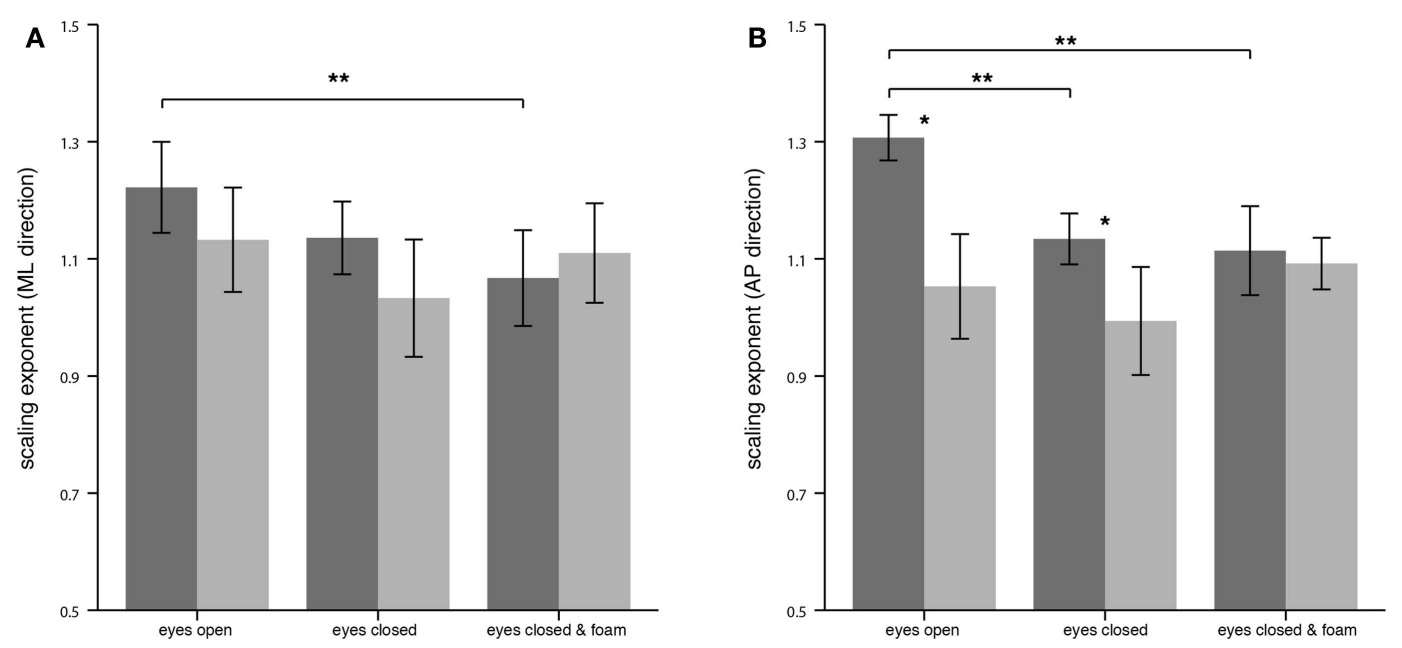

FIGURE 3 | Scaling exponent $\alpha$ of the center of pressure displacement in the medial-lateral (ML) direction (A) and in the anterior-posterior (AP) direction (B) for healthy controls $\left(\boldsymbol{n}=\mathbf{2 0}\right.$; dark gray) and patients $\left(\boldsymbol{n}=\mathbf{2 0}\right.$; light gray). Asterisks denote significant differences: $\left({ }^{*}\right)$ indicates $p<0.05,\left({ }^{*}\right)$ indicates $p<0.01$.
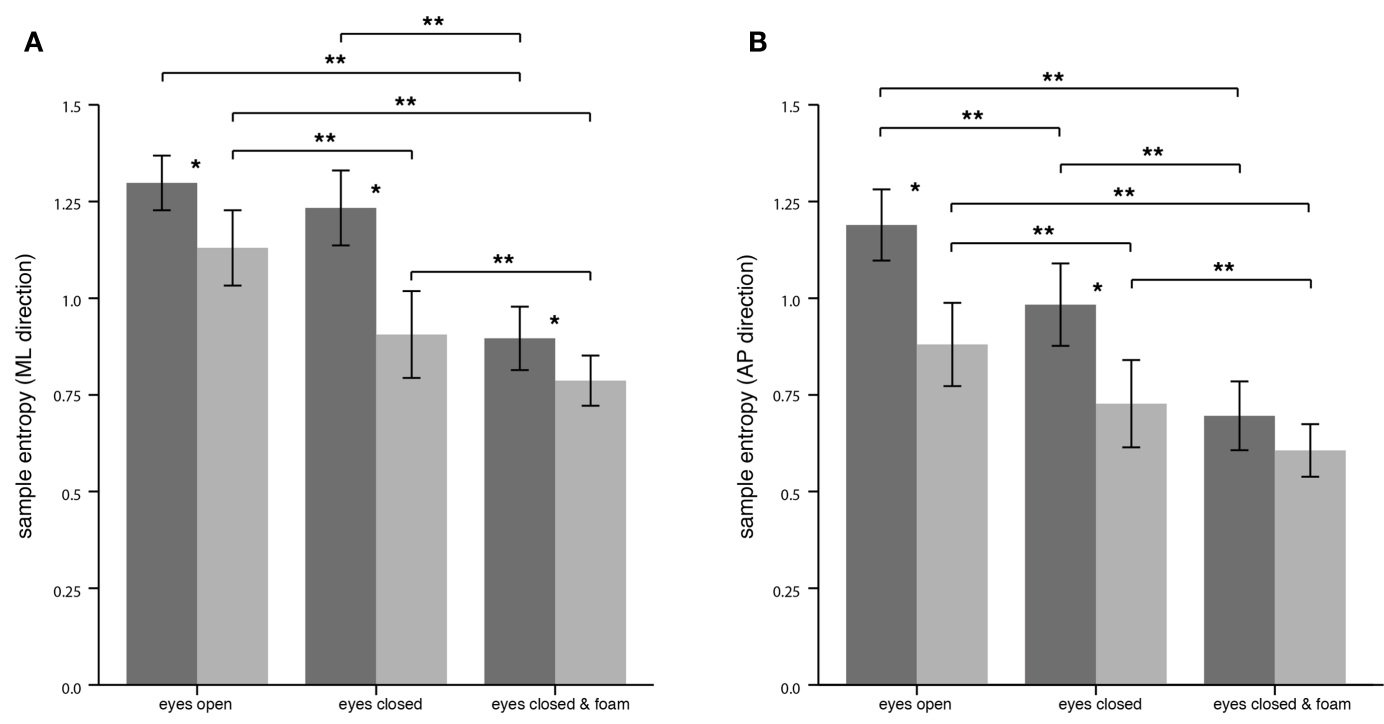

FIGURE 4 | Sample entropy of the center of pressure displacement in the medial-lateral (ML) direction (A) and in the anterior-posterior (AP) direction (B) for healthy controls ( $\boldsymbol{n}=\mathbf{2 0}$; dark gray) and patients $\left(\boldsymbol{n}=\mathbf{2 0}\right.$, light gray). Asterisks denote significant differences: $\left(^{*}\right)$ indicates $p<0.05,\left(^{* *}\right)$ indicates $p<0.01$.

only for the most demanding balance tasks. The observation that postural behavior in PPV converges to the healthy postural pattern for more demanding balance tasks has been previously described as a characteristic feature of somatoform dizziness syndromes (9).

Sample entropy, which describes the irregularity of the sway pattern, was found to be reduced in patients with PPV for normal stance condition, indicating that the dynamic structure of the posturogram is characterized by a higher regularity (13, 28). Decreased complexity in terms of a more regular sway pattern indicates that the postural behavior is more rigid within repeating patterns thereby loosing adaptability and local stability (13). Increasing the difficulty of the balance task led to a decrease in SEn in HS in accordance to previous studies (14, 32). This decrease was less pronounced in PPV; thereby SEn was similar between patients and controls for demanding tasks (9). Interestingly, a previous study investigating complexity of postural control in young and older adults found changes of the scaling exponent $\alpha$ and the entropy of sway toward different directions (15). While $\alpha$ was closer to $1 / f$ noise in the 
elderly, entropy was found to be increased compared to young adults. However, the authors concluded that the entropy results are inconsistent with the generally acknowledged hypothesis that complexity in the human physiological system decreases with aging $(33,37)$.

\section{POSTURAL CONTROL STRATEGIES OF PATIENTS WITH PPV}

Previous studies have proposed a relation between the regularity of CoP displacements and the amount of attention spent in the postural control $(13,14,35,38)$. According to this theory the regularity of body sway is positively correlated to the attentional effort (39). The altered postural control dynamics in PPV correspond to the patient's feeling of instability even on firm ground (19). In previous studies on PPV, it has been hypothesized that increased co-contraction of anti-gravity muscles may cause the altered sway pattern of the patients (20). HS also use co-contraction instead of reciprocal muscle activation when learning a new motor task or when uncertainty exists about the required motor response (40, 41). Co-contraction stabilizes joints and reduces their mechanical sensitivity to external perturbations and thereby shortens the settling time of the system (40). The changes in variability, which we found in PPV, might be compatible with the co-contraction hypothesis (42), but further investigations including EMG recordings of anti-gravity muscles have to be conducted. Our findings might suggest that inappropriate postural control in PPV is caused by a shift of the postural strategy to a more attentional control. The accompanying decrease of complexity might hinder appropriate

\section{REFERENCES}

1. Brandt T. Phobic postural vertigo. Neurology (1996) 46(6):15159. doi:10.1212/WNL.46.6.1515

2. Best C, Eckhardt-Henn A, Diener G, Bense S, Breuer P, Dieterich M. Interaction of somatoform and vestibular disorders. $J \mathrm{Neu}$ rol Neurosurg Psychiatr (2006) 77(5):658-64. doi:10.1136/jnnp. 2005.072934

3. Eckhardt-Henn A, Breuer P, Thomalske C, Hoffmann SO, Hopf HC. Anxiety disorders and other psychiatric subgroups in patients complaining of dizziness. J Anxiety Disord (2003) 17(4):369-88. doi: 10.1016/S0887-6185(02)00226-8

4. Furman JM, Jacob RG. Psychiatric dizziness. Neurology (1997) 48(5):1161-6. doi:10.1212/WNL.48.5.1161

5. Bronstein AM. Visual vertigo syndrome: clinical and posturography findings. J Neurol Neurosurg Psychiatr (1995) 59(5):472-6. doi:10. 1136/jnnp.59.5.472

6. Staab JP, Ruckenstein MJ. Expanding the differential diagnosis of chronic dizziness. Arch Otolaryngol Head Neck Surg (2007) 133(2):170-6. doi:10.1001/ archotol.133.2.170

7. Brandt T, Huppert D, Dieterich M. Phobic postural vertigo: a first follow-up. $J \quad \mathrm{Neu}$ rol (1994) 241(4):191-5. doi:10.1007/BF00863767

8. Krafczyk S, Tietze S, Swoboda W, Valkovic P, Brandt T. Artificial neural network: a new diagnostic posturographic tool for disorders of stance. Clin Neurophysiol (2006) 117(8):1692-8. doi:10.1016/ j.clinph.2006.04.022

9. Querner V, Krafczyk S, Dieterich M, Brandt T. Patients with somatoform phobic postural vertigo: the more difficult the balance task, the better the balance performance. Neurosci Lett (2000) 285(1):21-4. doi: 10.1016/S0304-3940(00)01008-9

10. Latash ML, Scholz JP, Schoner G. Motor control strategies revealed in the structure of motor variability. Exerc Sport Sci Rev (2002) 30(1):26-31. doi:10.1097/ 00003677-200201000-00006

11. Blaszczyk JW, Klonowski W. Postural stability and fractal dynamics. 61(2):105-12.

12. Collins JJ, De Luca CJ. Random walking during quiet standing. Phys Rev Lett (1994) 73(5):764-7. doi:10. 1103/PhysRevLett.73.764

13. Borg FG, Laxaback G. Entropy of roeng Rehabil (2010) 7:38. doi:10. 1186/1743-0003-7-38 Acta Neurobiol Exp (Wars) (2001) balance-some recent results. J Neu-

reactive tuning thereby causing inadequate responses to perturbation (33). In HS similar postural control modes are observed only under demanding balance conditions, like standing on ice or on uneven surfaces.

Studies on the clinical course of PPV have shown the reversibility of the subjective symptoms (19). For stroke patients it has been shown that the regularity of CoP fluctuations decreases during the rehabilitation whereas in parallel the postural stability increases (35). Analysis of CoP fluctuations might be a promising tool for measuring an objective improvement during recovery from PPV. For the future it will be of interest to investigate the sensitivity and specificity of alterations in the scaling exponent $\alpha$ and SEn as a diagnostic tool in PPV.

In conclusion, the results of the present study indicate an impaired postural behavior of patients with PPV predominantly under normal balance conditions. Their balance control appears to be more regular and constrained compared to HS. These changes in balance control might be linked to an anxious control of posture. Analysis of nonlinearities in body sway carries the potential to achieve objective measurements for the diagnosis of PPV.

\section{ACKNOWLEDGMENTS}

The authors thank Judy Benson for copy-editing the article. The work was supported by the German research foundation (Deutsche Forschungsgemeinschaft, DFG JA 1087/1-1), the German Hertie Foundation, and the Federal Ministry for Education and Science of Germany (BMBF 01EO0901).

14. Donker SF, Roerdink M, Greven AJ, Beek PJ. Regularity of centerof-pressure trajectories depends on the amount of attention invested in postural control. Exp Brain Res (2007) 181(1):1-11. doi:10.1007/ s00221-007-0905-4

15. Duarte M, Sternad D. Complexity of human postural control in young and older adults during prolonged standing. Exp Brain Res (2008) 191(3):265-76. doi:10.1007/ s00221-008-1521-7

16. Madeleine P, Nielsen M, ArendtNielsen L. Characterization of postural control deficit in whiplash patients by means of linear and nonlinear analyses - a pilot study. J Electromyogr Kinesiol (2011) 21(2):2917. doi:10.1016/j.jelekin.2010.05.006

17. Doyle TL, Newton RU, Burnett AF. Reliability of traditional and fractal dimension measures of quiet stance center of pressure in young, healthy people. Arch Phys Med Rehabil (2005) 86(10):2034-40. doi:10. 1016/j.apmr.2005.05.014

18. Holmberg J, Tjernstrom F, Karlberg M, Fransson PA, Magnusson M. Reduced postural differences between phobic postural vertigo patients and healthy subjects during a postural threat. $J \mathrm{Neu}$ rol (2009) 256(8):1258-62. doi:10. 1007/s00415-009-5110-x
19. Kapfhammer HP, Mayer C, Hock U, Huppert D, Dieterich M, Brandt T. Course of illness in phobic postural vertigo. Acta Neurol Scand (1997) 95(1):23-8. doi:10.1111/j. 1600-0404.1997.tb00063.x

20. Krafczyk S, Schlamp V, Dieterich M, Haberhauer P, Brandt T. Increased body sway at $3.5-8 \mathrm{~Hz}$ in patients with phobic postural vertigo. Neurosci Lett (1999) 259(3):149 52. doi:10.1016/S0304-3940(98) 00917-3

21. Woollacott M, Shumway-Cook A. Attention and the control of posture and gait: a review of an emerging area of research. Gait Posture (2002) 16(1):1-14. doi:10. 1016/S0966-6362(01)00156-4

22. Jordan K, Challis JH, Newell KM. Walking speed influences on gait cycle variability. Gait Posture (2007) 26(1):128-34. doi:10.1016/j. gaitpost.2006.08.010

23. Halmagyi GM, Curthoys IS. A clinical sign of canal paresis. Arch Neurol (1988) 45(7):7379. doi:10.1001/archneur.1988. 00520310043015

24. Peng CK, Havlin S, Stanley HE, Goldberger AL. Quantification of scaling exponents and crossover phenomena in nonstationary heartbeat time series. Chaos (1995) 5(1):82-7. doi:10.1063/1.166141 
25. Delignieres D, Torre K, Lemoine L. Methodological issues in the application of monofractal analyses in psychological and behavioral research. Nonlinear Dynamics Psychol Life Sci (2005) 9(4):435-61.

26. Fossion R, Landa E, Stránský P, Velazquez V, Vieyra JL, Garduno I, et al. Scale invariance as a symmetry in physical and biological systems: listening to photons, bubbles and heartbeats. AIP Conf Proc (2010) 2010:74. doi:10.1063/1.3537868

27. Keshner MS. 1/f noise. Proc IEEE (1982) 70(3):212-8. doi:10.1109/ PROC.1982.12282

28. Lake DE, Richman JS, Griffin MP, Moorman JR. Sample entropy analysis of neonatal heart rate variability. Am J Physiol Regul Integr Comp Physiol (2002) 283(3):R78997.

29. Richman JS, Moorman JR. Physiological time-series analysis using approximate entropy and sample entropy. Am J Physiol Heart Circ Physiol (2000) 278(6):H2039-49.

30. Govindan R, Wilson J, Eswaran H, Lowery C, Preißl H. Revisiting sample entropy analysis. Physica A (2007) 376:158-64. doi:10.1016/j. physa.2006.10.077

31. Goldberger AL, Amaral LA, Glass L, Hausdorff JM, Ivanov PC, Mark RG, et al. PhysioBank, PhysioToolkit, and PhysioNet: components of a new research resource for complex physiologic signals. Circulation (2000) 101(23):e215-20. doi: 10.1161/01.CIR.101.23.e215

32. Ramdani S, Seigle B, Lagarde J, Bouchara F, Bernard PL. On the use of sample entropy to analyze human postural sway data. Med Eng Phys (2009) 31(8):1023-31. doi:10.1016/ j.medengphy.2009.06.004

33. Lipsitz LA. Dynamics of stability: the physiologic basis of functional health and frailty. J Gerontol A Biol Sci Med Sci (2002) 57(3):B115-25. doi:10.1093/gerona/57.3.B115

34. Vaillancourt DE, Newell KM. Changing complexity in human behavior and physiology through aging and disease. Neurobiol Aging (2002) 23(1):1-11. doi:10.1016/ S0197-4580(01)00247-0

35. Roerdink M, De Haart M, Daffertshofer A, Donker SF, Geurts AC, Beek PJ. Dynamical structure of center-of-pressure trajectories in patients recovering from stroke. Exp Brain Res (2006) 174(2):256-69. doi:10.1007/ s00221-006-0441-7

36. Slifkin AB, Newell KM. Noise, information transmission, and force variability. J Exp Psychol Hum Percept Perform (1999) 25(3):837-51. doi:10.1037/0096-1523.25.3.837
37. Goldberger AL, Peng C, Lipsitz LA. What is physiologic complexity and how does it change with aging and disease? Neurobiol Aging (2002) 23(1):23-6. doi:10. 1016/S0197-4580(01)00266-4

38. Roerdink M, Hlavackova P, Vuillerme N. Center-of-pressure regularity as a marker for attentional investment in postural control: a comparison between sitting and standing postures. Hum Mov Sci (2011) 30(2):203-12. doi:10.1016/j.humov.2010.04.005

39. Goldberger AL, Amaral LA, Hausdorff JM, Ivanov P, Peng CK, Stanley HE. Fractal dynamics in physiology: alterations with disease and aging. Proc Natl Acad Sci U S A (2002) 99(Suppl 1):2466-72. doi:10.1073/ pnas.012579499

40. De Luca CJ, Mambrito B. Voluntary control of motor units in human antagonist muscles: coactivation and reciprocal activation. $J$ Neurophysiol (1987) 58(3):525-42.

41. Smith AM. The coactivation of antagonist muscles. Can J Physiol Pharmacol (1981) 59(7):733-47. doi:10.1139/y81-110

42. Laughton CA, Slavin M, Katdare K, Nolan L, Bean JF, Kerrigan DC, et al. Aging, muscle activity, and balance control: physiologic changes associated with balance impairment. Gait
Posture (2003) 18(2):101-8. doi:10. 1016/S0966-6362(02)00200-X

Conflict of Interest Statement: The authors declare that the research was conducted in the absence of any commercial or financial relationships that could be construed as a potential conflict of interest.

Received: 18 March 2013; accepted: 29 July 2013; published online: 15 August 2013.

Citation: Schniepp R, Wuehr M, Pradhan C, Novozhilov S, Krafczyk S, Brandt T and Jahn K (2013) Nonlinear variability of body sway in patients with phobic postural vertigo. Front. Neurol. 4:115. doi: 10.3389/fneur.2013.00115

This article was submitted to Frontiers in Neuro-otology, a specialty of Frontiers in Neurology.

Copyright (c) 2013 Schniepp, Wuehr, Pradhan, Novozhilov, Krafczyk, Brandt and Jahn. This is an open-access article distributed under the terms of the Creative Commons Attribution License (CC BY). The use, distribution or reproduction in other forums is permitted, provided the original author(s) or licensor are credited and that the original publication in this journal is cited, in accordance with accepted academic practice. No use, distribution or reproduction is permitted which does not comply with these terms. 